\title{
Early and late pulmonary manifestations of COVID-19 on computed tomography scan
}

\author{
Samarra Mongi Kaabi ${ }^{1 *}$, Ahmad Nasser Madkhali ${ }^{2}$, Naif Hussain Alqahtani ${ }^{3}$, \\ Ammar Adel Bakhsh ${ }^{4}$, Yousef Hussain Alharthi ${ }^{5}$, Nawal Misfer Alwadai ${ }^{6}$, \\ Abdullah Meshaan Alotaibi ${ }^{7}$, Saad Obaid Alotaibi ${ }^{8}$, Ahmed Abdualrhman Aljohani', \\ Sarah Thamer Aldarsouny ${ }^{10}$, Doha Jamal Ahmad ${ }^{11}$
}

\author{
${ }^{1}$ Department of Radiology, International Medical Center, Jeddah, Saudi Arabia \\ ${ }^{2}$ College of Medicine, Ibn Sina National College, Jeddah, Saudi Arabia \\ ${ }^{3}$ Department of Radiology, King Abdulaziz Specialist Hospital, Taif, Saudi Arabia \\ ${ }^{4}$ College of Medicine, Umm Al-Qura University, Mecca, Saudi Arabia \\ ${ }^{5}$ College of Medicine, University of Tabuk, Tabuk, Saudi Arabia \\ ${ }^{6}$ Department of Internal Medicine, Prince Sultan Military Medical City, Riyadh, Saudi Arabia \\ ${ }^{7}$ Department of Radiology, King Fahad Armed Forces Hospital, Jeddah, Saudi Arabia \\ ${ }^{8}$ Department of Radiology, Alhada Military Hospital, Taif, Saudi Arabia \\ ${ }^{9}$ College of Medicine, Taibah University, Medina, Saudi Arabia \\ ${ }^{10}$ College of Medicine, Almaarefa University, Riyadh, Saudi Arabia \\ ${ }^{11}$ Department of Radiology, Al Aziziah Children Hospital, Jeddah, Saudi Arabia
}

Received: 21 June 2021

Accepted: 15 July 2021

\section{*Correspondence:}

Dr. Samarra Mongi Kaabi,

E-mail: samarrakaabi@gmail.com

Copyright: (C) the author(s), publisher and licensee Medip Academy. This is an open-access article distributed under the terms of the Creative Commons Attribution Non-Commercial License, which permits unrestricted non-commercial use, distribution, and reproduction in any medium, provided the original work is properly cited.

\begin{abstract}
Computed tomography (CT) of the chest has been previously reported as an efficacious approach for the early diagnosis and suspicion of COVID-19 infection, as diagnosis with other modalities is usually time-consuming and cannot detect the disease within the early stages. Many pulmonary manifestations have been previously observed under CT imaging of the chest. All of them have been linked with the different stages of the disease, indicating their abilities to diagnose and follow-up these patients. This present literature review aims to discuss the role of CT imaging of the chest in COVID-19 infections and it also aims to elaborate the common pulmonary manifestations that can be frequently observed to help with the diagnosis and prognosis of the disease. Moreover, ground-glass opacities (GGOs) have been reported among studies in the literature to be the most common findings as they remain the earliest among other diseases. The literature review also aims to invistagate whether GGOs can fade away or progress to consolidate in order to decide the prognosis of the disease and to identify the severity of cases. Consolidations have also been previously stated among studies in the literature as frequent pulmonary manifestations affecting patients with COVID-19 infections and are estimated to be present in 2-63\% of patients with COVID-19 infections. Other pulmonary manifestations might also include bronchiectasis, lymphadenopathy, nodules, pleural effusion or thickening, and halo sign.
\end{abstract}

Keywords: COVID-19, Pulmonary, CT, Diagnosis, Prognosis 


\section{INTRODUCTION}

COVID-19 is a disease that caused by the severe acute respiratory syndrome coronavirus 2 (SARS-CoV-2). It was first reported in China, emerging from a wet market in Wuhan, specifically, in late 2019. The progression of the disease has become significant in the following months, as it has nearly affected almost all countries over the world with millions of cases and deaths globally. ${ }^{1,2}$ The impact of the disease is not only confined to the health status of the affected patients, but it also related to the social, economic, and scientific aspects of population all over the world. ${ }^{3}$ Many management approaches have been proposed for the adequate prevention and treatment of the disease. However, none of the previously suggested modalities was found to be efficacious in managing the development of the disease. Accordingly, efforts were directed to identify the infected cases as early as possible and isolate them to adequately contain the rates of infections and reduce the spread of the infection.

The clinical presentation for COVID-19 patients is hugely variable and even be asymptomatic. ${ }^{4}$ The symptoms might include illnesses, such as flu, headache, fever, diarrhea, cough, sore throat, and other symptoms. ${ }^{4}$ However, the COVID-19 disease can be severe in some cases where there might be severe organ damage leading to some complications related to the lungs, kidney, liver, and central nervous system. ${ }^{5-10}$ Moreover, pulmonary manifestations remain the most common manifestations that can be observed among these patients and some studies have reported that the pulmonary findings can be used to diagnose or suspect the infection, and predict the prognosis of the disease. ${ }^{7} \mathrm{CT}$ of the chest has been previously reported as an efficacious approach for the early diagnosis and suspicion of COVID-19 infection, as diagnosis with other modalities is usually timeconsuming and cannot detect the disease within the early stages. CT can be used to indicate the infection, especially in patients with severe pulmonary-related symptoms, as such cough, dyspnea, and fever. It is also recommended to follow-up with the previously indicated patients for disease resolution. ${ }^{11,12}$

Many pulmonary manifestations have been previously observed under CT imaging of the chest, which have been linked with the different stages of the disease, indicating their abilities to diagnose and follow-up these patients. ${ }^{13}$ The present literature review aims to discuss the previously reported pulmonary manifestations that are usually observed during early and late COVID-19 infections.

This extensive literature search gathered data from the Medline, Cochrane, and Embase databases on $10^{\text {th }}$ May 2021 using the medical subject headings (MeSH) and a combination of all possible related terms. This was followed by the manual search for papers in Google scholar and the reference lists of the initially included papers. ${ }^{14,15}$ Papers discussing pulmonary manifestations of COVID-19 on CT scan were screened for relevant information and we posed no limits on date, language, age of participants, or publication type.

\section{DISCUSSION}

\section{Importance of conducting $C T$ chest imaging for COVID-19 patients}

CT scanning has been previously reported as an efficacious modality for the early diagnosis and follow-up of COVID-19 infections, according to many previous observational investigations which reported the definitive presence of COVID-19 related pathological features. Moreover, CT scanning has been previously reported as the most standardized test modality for indicating the infection as the most specific modality , and real-time reverse-transcription polymerase chain reaction (RTPCR) has been stated by other investigations that the technique should not be used alone in diagnosing the infection and other modalities should be approached due to the very low estimated sensitivity rates for this technique. ${ }^{16}$ Accordingly, CT imaging of the chest was suggested because of the estimated higher sensitivity rate. ${ }^{17}$ The main advantages of CT use over RT-PCR are that CT imaging is fast, widely available, and noninvasive. ${ }^{17}$ Based on these recommendations, RT-PCR and CT scan of the chest have been reported to be used concomitantly for diagnosing COVID-19 infections. ${ }^{11}$ Although CT imaging of the chest has been reported to have a very high sensitivity rate in detecting any potential underlying pathology, the estimated specificity for the same modality has been reported to be very low. ${ }^{18}$ Therefore, it has been reported that CT imaging might sometimes lead to false-negative diagnostic events as a result of the potential presence of overlapping other etiologies that might cause the same CT-related pathological findings. ${ }^{19,20}$ Besides, a previous investigation by $\mathrm{Ai}$ et al reported that follow-up for patients with COVID-19 infections was effectively able to detect significant improvement of the underlying pathology in $42 \%$ of their included population while RTPCR results for the same patients were still positive. ${ }^{18}$ According to many management and screening protocols for COVID-19 patients, it has been reported that CT imaging should be approached as an efficacious modality for the early diagnosis and follow-up for these patients to obtain better outcomes with estimated higher sensitivity and specificity rates for older patients and patients that are highly suspected to have COVID-19 infection. ${ }^{18,21,22}$

\section{Early diagnostic CT pulmonary manifestations}

Among the different studies in the literature, many pulmonary manifestations for COVID-19 patients have been observed using CT imaging of the chest (Figure 1). Among the reported manifestations, ground-glass opacities (GGOs) remain the most common among patients and may be considered the main pathology that is usually significantly associated with a positive prediction 
of the infection. ${ }^{5,23}$ Additionally, it has been observed that the lesion usually affects the lateral parts of both lungs under the pleura. It has also been found that the pathology might extend to other central parts of the lung when the lesion might be extensive. ${ }^{24}$ Moreover, a previous investigation by Bernheim et al previously reported that the left lower lobe is usually more affected than the upper, middle, and right lobes. ${ }^{24}$ However, it has also been observed that the lesion does not usually last alongside the course of the disease and usually converts to consolidation or fibrosis as the disease progresses. A previous investigation by $\mathrm{Pan}$ et al also reported that GGOs were the most predominant lung pathology that was observed during the early stages of the disease in their COVID-19 patients; however, patients with good prognosis resolution of these opacities were frequently observed and GGOs converted into crazy-paving and consolidation lesions within the first two weeks from the onset of symptoms. ${ }^{13}$ Moreover, in a case report study by $\mathrm{Xu}$ et al, the authors reported that in a postmortem investigation of a patient that died from severe COVID19 infection. ${ }^{25}$ The main underlying pathology that was noticed in this patient was GGOs, which was indicated by the presence of pulmonary edema. Moreover, other reports also showed that in such cases, consolidation and interstitial thickening can be observed in some cases together with GGOs, which are all suggestive of the presence of pneumonia and the presence of underlying lung injury. ${ }^{26,27}$

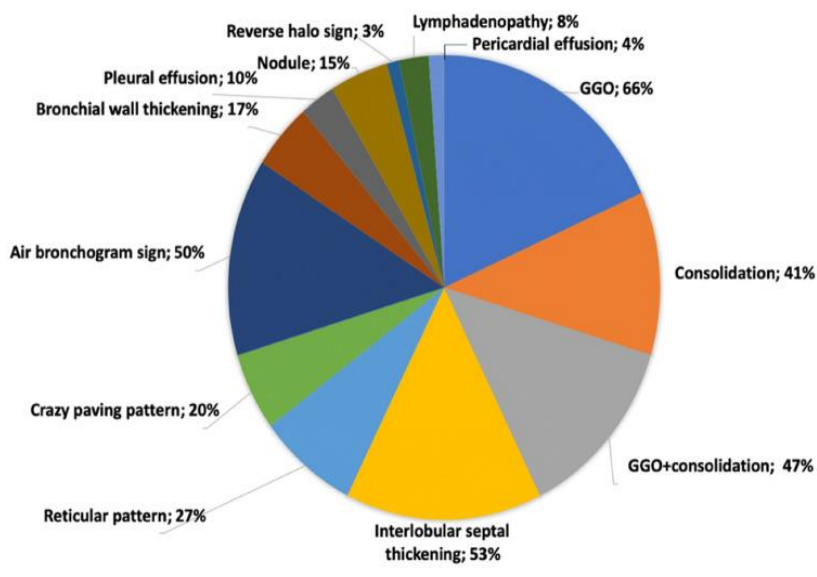

Figure 1: CT pulmonary manifestations of COVID-19 patients and the estimated percentage for each, according to previous investigations. ${ }^{39}$

Consolidations have also been previously reported among studies in the literature as frequent pulmonary manifestations affecting patients with COVID-19 infections and are estimated to be present in $2-63 \%$ of patients with COVID-19 infections. ${ }^{28,29}$ The pattern of these manifestations has also been reported to be variable among patients, being patchy, multifocal, or segmental, and might have a peri-bronchovascular or sub-pleural distribution. Some investigations reported that the interpretation of the presence of consolidations should be carefully approached as they might be indicative of severe COVID-19 disease, as they usually occur among patients that are over 50 years of age and during the second weeks from the disease progression. ${ }^{13,27,30}$ Lymphocyte infiltration can also be present in patients with COVID-19 infections and can eventually lead to the development of reticular opacities, which has been observed among studies in the literature to be the third most common pulmonary manifestation that can be observed among COVID-19 patients as they usually progress alongside the course of the disease. ${ }^{25,28}$ Although crazy-pavings can be observed in $5-36 \%$ of patients with COVID-19 infections, previous investigations have reported that these findings are less frequent to be observed than consolidations and GGOs. ${ }^{13,29}$ On the other hand, the presence of this manifestation can be used to predict the start of the peak stage when they have been observed alongside consolidation and GGOs. ${ }^{13}$ Air bronchograms have also been previously observed among COVID-19 patients. ${ }^{27,31}$ Other pulmonary manifestations might also include bronchiectasis, lymphadenopathy, nodules, pleural effusion or thickening, and halo sign. ${ }^{13,17,28,30,32}$ Furthermore, previous investigations have stated that some CT pulmonary manifestations might be significant predictors for having severe COVID-19 disease. For instance, Yuan et al previously reported that the presence of consolidation and a high median CT score were significantly associated with death among patients who died during hospitalization as compared to other patients who did not die or were discharged. ${ }^{33}$ Old age and the presence of underlying comorbidities have also been previously reported as significant predictors of mortality among these patients. ${ }^{34}$ These findings were also indicated by $\mathrm{Li}$ et al, that also reported that linear opacities, crazy-pavings, and consolidation were all significantly associated with the development of severe COVID-19 disease. ${ }^{29}$

\section{Late prognostic CT pulmonary manifestations}

Evidence from the current literature also showed that CT imaging of the chest is important to evaluate the prognosis of COVID-19 infections. It has been reported that pathological pulmonary manifestations usually change over time since the time of the infection and until healing occurs. For instance, a previous investigation by Pan et al tracked the pulmonary changes during COVID19 infections to find whether the pathology changes overtime or not. ${ }^{13}$ The authors have finally concluded that COVID-19 related pathology can be divided into four stages including the early, progressive, peak, and absorption stages which usually compromise the following time intervals: $0-4,5-8,9-13$, and $\geq 14$ days, respectively. Moreover, the authors of the same study also reported that they visualized the five lobes of the lung and put a scoring system from 0 to 5 , based on the severity and involvement of the different parts of the affected lungs. No involvement was recorded as $0,<5 \%$ involvement was recorded as $1,25 \%$ involvement scored 2, 26-49\% involvement scored 3, 50-75\% involvement scored 4 , while $>75 \%$ involvement scored 5 . The total of 
the estimated CT scores were then used to process and evaluate the severity of the underlying pathology, as they reported that 0 denotes or minimal injury while a maximum score of 25 denoted severe underlying pathology. Moreover, the authors reported that the observed pulmonary manifestations usually progress from the onset of symptoms until 10 days when the pathology usually peaks, and then the severity and observed demarcations usually decrease. The previous investigation by Yuan et al also reported that severe COVID-19 disease was associated with a CT score cutoff point of 24.5 , and the authors reported that the specificity and sensitivity for this point to predict mortality among these patients were $84.5 \%$ and $85.6 \%$, respectively. ${ }^{33}$ Previous investigations have also reported that GGOs were observed to more a well-formed characteristic of stage 2 of lung pathology during COVID-19 infections, and crazy-waving patterns were the most common to be seen during this stage. ${ }^{35}$ Moreover, it has been stated that consolidation is usually the underlying pathology with reduced observations of GGOs during the third stage since the infection occurred. A previous investigation by Salehi et al reported that among their population of 919 COVID-19 patients, GGOs were frequent during the intermittent stage and were frequently seen while during the following progressive stage, multifocal consolidation, crazy-paving patterns, and thickening of the related lung septa were more frequent than the previously observed GGOs ${ }^{35}$ In general, previous investigations have reported that such observations are not specific to COVID-19 infections only, as pneumonia usually develops following other infections, radiation, administration of certain drugs, and transition from GGOs to consolidation is a significant marking for the development of pneumonia. ${ }^{36}$ The same findings were also reported in a previous longitudinal follow-up investigation by Wang et al. ${ }^{37}$ which showed that GGOs were purely observed as the main and maybe the only pulmonary manifestation among their patients, which also significantly changed to a mix of GGOs and linear opacities due to consolidation within the days of the third stage. Pulmonary embolism should also be suspected in cases of severe infections, and assessment of D-dimer levels might help with establishing a proper diagnosis for early and adequate management. Considering contrast-enhanced CT has also been previously recommended in such cases; however, precautions should be considered in patients with severe infections and underlying renal diseases. ${ }^{38}$

\section{CONCLUSION}

In the present literature review, it has discussed the role of CT imaging of the chest in COVID-19 infections, in addition to elaborating the common pulmonary manifestations that can be frequently observed and can help with the diagnosis and prognosis of the disease. GGOs and consolidations have been reported among studies in the literature to be the most common findings, and GGOs remain the earliest. Whether GGOs can fade away or progress to consolidations can also decide the prognosis of the disease and identify severe cases.

Funding: No funding sources

Conflict of interest: None declared

Ethical approval: Not required

\section{REFERENCES}

1. Zhu N, Zhang D, Wang W. A Novel Coronavirus from Patients with Pneumonia in China, 2019. N England j med. 2020;382(8):727-33.

2. Sohrabi C, Alsafi Z, O'Neill N. World Health Organization declares global emergency: A review of the 2019 novel coronavirus (COVID-19). Int $\mathrm{j}$ surgery. 2020;76:71-6.

3. Ghozy S, Abdelaal A, Shah J, Parker KE, Islam SMS. COVID-19 and physical inactivity: Teetering on the edge of a deadlier pandemic? J Glob Health. 2021;11:03031.

4. Elgamasy S, Sakr EE, Kamel MG. Epidemiologic features and clinical course of COVID-19: a retrospective analysis of 19 patients in Germany. Future Virol. 2021;2020-256.

5. Wang D, Hu B, Hu C. Clinical Characteristics of 138 Hospitalized Patients With 2019 Novel CoronavirusInfected Pneumonia in Wuhan, China. Jama. 2020;323(11):1061-9.

6. Gupta A, Madhavan MV, Sehgal K. Extrapulmonary manifestations of COVID-19. Nature Medi. 2020;26(7):1017-32.

7. Johnson KD, Harris C, Cain JK, Hummer C, Goyal $\mathrm{H}$, Perisetti A. Pulmonary and Extra-Pulmonary Clinical Manifestations of COVID-19. Front Med. 2020;7:526-6.

8. Van Cuong L, Giang HTN, Linh LK. The first Vietnamese case of COVID-19 acquired from China. Lancet Infect Dis. 2020;20(4):408-9.

9. Abdelaziz NA, Ghozy S, Salah A, Abbas AR, ElQushayri AE, Islam SMS. Full recovery of a patient with COVID-19-induced acute kidney injury. Eur J Med Case Rep. 2021;5(1):26-30.

10. Elgamasy S, Kamel MG, Ghozy S, Khalil A, Morra ME, Islam SMS. First case of focal epilepsy associated with SARS-coronavirus-2. J Med Virol. 2020;92(10):2238-42.

11. Xie X, Zhong Z, Zhao W, Zheng C, Wang F, Liu J. Chest CT for Typical Coronavirus Disease 2019 (COVID-19) Pneumonia: Relationship to Negative RT-PCR Testing. Radiology. 2020;296(2):E41-5.

12. Feng H, Liu Y, Lv M, Zhong J. A case report of COVID-19 with false negative RT-PCR test: necessity of chest CT. Japanese $\mathrm{j}$ radiol. 2020;38(5):409-10.

13. Pan F, Ye T, Sun P. Time Course of Lung Changes at Chest CT during Recovery from Coronavirus Disease 2019 (COVID-19). Radiology. 2020;295(3):715-21.

14. Hashan MR, Ghozy S, El-Qushayri AE, Pial RH, Hossain MA, Al Kibria GM. Association of dengue disease severity and blood group: A systematic 
review and meta-analysis. Rev Med Virol. 2021;31(1):1-9.

15. El-Qushayri AE, Ghozy S, Abbas AS. Hyperimmunoglobulin therapy for the prevention and treatment of congenital cytomegalovirus: a systematic review and meta-analysis. Expert Rev Anti Infect Ther. 2020:1-9.

16. Fang Y, Zhang H, Xie J. Sensitivity of Chest CT for COVID-19: Comparison to RT-PCR. Radiology. 2020;296(2):E115-7.

17. Fang Y, Zhang H, Xu Y, Xie J, Pang P, Ji W. CT Manifestations of Two Cases of 2019 Novel Coronavirus (2019-nCoV) Pneumonia. Radiol. 2020;295(1):208-9.

18. Ai T, Yang Z, Hou H. Correlation of Chest CT and RT-PCR Testing for Coronavirus Disease 2019 (COVID-19) in China: A Report of 1014 Cases. Radiology. 2020;296(2):E32-40.

19. Bai HX, Hsieh B, Xiong Z. Performance of Radiologists in Differentiating COVID-19 from NonCOVID-19 Viral Pneumonia at Chest CT. Radiology. 2020;296(2):E46-54.

20. Liu M, Zeng W, Wen Y, Zheng Y, Lv F, Xiao K. COVID-19 pneumonia: CT findings of 122 patients and differentiation from influenza pneumonia. Eur radiol. 2020;30(10):5463-9.

21. Rubin GD, Ryerson CJ, Haramati LB. The Role of Chest Imaging in Patient Management during the COVID-19 Pandemic: A Multinational Consensus Statement from the Fleischner Society. Radiology. 2020;296(1):172-80.

22. Li Y, Xia L. Coronavirus Disease 2019 (COVID-19): Role of Chest CT in Diagnosis and Management. AJR Am j roentgenol. 2020;214(6):1280-6.

23. Ye Z, Zhang Y, Wang Y, Huang Z, Song B. Chest CT manifestations of new coronavirus disease 2019 (COVID-19): a pictorial review. Eur radiol. 2020;30(8):4381-9.

24. Bernheim A, Mei X, Huang M. Chest CT Findings in Coronavirus Disease-19 (COVID-19): Relationship to Duration of Infection. Radiology. 2020;295(3):200463.

25. $\mathrm{Xu} \mathrm{Z}$, Shi L, Wang Y. Pathological findings of COVID-19 associated with acute respiratory distress syndrome. Lancet Respiratory med. 2020;8(4):42022.

26. Kanne JP. Chest CT Findings in 2019 Novel Coronavirus (2019-nCoV) Infections from Wuhan, China: Key Points for the Radiologist. Radiology. 2020;295(1):16-7.

27. Song F, Shi N, Shan F. Emerging 2019 Novel Coronavirus (2019-nCoV) Pneumonia. Radiology. 2020;295(1):210-17.

28. Wu J, Wu X, Zeng W. Chest CT Findings in Patients With Coronavirus Disease 2019 and Its Relationship
With Clinical Features. Investigative radiol. 2020;55(5):257-61.

29. Li K, Wu J, Wu F. The Clinical and Chest CT Features Associated With Severe and Critical COVID-19 Pneumonia. Investigative radiol. 2020;55(6):327-31.

30. Shi H, Han X, Jiang N. Radiological findings from 81 patients with COVID-19 pneumonia in Wuhan, China: a descriptive study. Lancet Infectious dis. 2020;20(4):425-34.

31. Liu RX, Guoqiang Q, Wang Y. A observational autopsy report of COVID-19 (Chinese). J Forensic Med. 2020;36:19-21.

32. Li X, Zeng X, Liu B, Yu Y. COVID-19 infection presenting with CT halo sign. Radiology: Cardiothoracic Imaging. 2020;2(1):e200026.

33. Yuan M, Yin W, Tao Z, Tan W, Hu Y. Association of radiologic findings with mortality of patients infected with 2019 novel coronavirus in Wuhan, China. PloS one. 2020;15(3):e0230548.

34. Yang W, Cao Q, Qin L, et al. Clinical characteristics and imaging manifestations of the 2019 novel coronavirus disease (COVID-19):A multi-center study in Wenzhou city, Zhejiang, China. J infect. 2020;80(4):388-93.

35. Salehi S, Abedi A, Balakrishnan S, Gholamrezanezhad A. Coronavirus Disease 2019 (COVID-19): A Systematic Review of Imaging Findings in 919 Patients. AJR Am j roentgenol. 2020;215(1):87-93.

36. Kligerman SJ, Franks TJ, Galvin JR. From the radiologic pathology archives: organization and fibrosis as a response to lung injury in diffuse alveolar damage, organizing pneumonia, and acute fibrinous and organizing pneumonia. Radiographics: a review publication of the Radiological Society of North America, Inc. 2013;33(7):1951-75.

37. Wang Y, Dong C, Hu Y. Temporal Changes of CT Findings in 90 Patients with COVID-19 Pneumonia: A Longitudinal Study. Radiology. 2020;296(2):E5564.

38. Tang N, Li D, Wang X, Sun Z. Abnormal coagulation parameters are associated with poor prognosis in patients with novel coronavirus pneumonia. $\mathrm{J}$ thrombosis haemostasis. 2020;18(4):844-7.

39. Carotti M, Salaffi F, Sarzi-Puttini P. Chest CT features of coronavirus disease 2019 (COVID-19) pneumonia: key points for radiologists. Radiol Med. 2020;125(7):636-46.

Cite this article as: Kaabi SM, Madkhali AN, Alqahtani NH, Bakhsh AA, Alharthi YH, Alwadai $\mathrm{NM}$ et al. Early and late pulmonary manifestations of COVID-19 on computed tomography scan. Int J Community Med Public Health 2021;8:4102-6. 